\title{
CONSERVAÇÃO DE ESPÉCIES AMEAÇADAS DO RIO GRANDE DO SUL ATRAVÉS DE SEU USO NO PAISAGISMO
}

\author{
Juliana Gonçalves Silva ${ }^{1}$ e Luís Fernando Carvalho Perelló ${ }^{2}$
}

(recebido em 22.09.2010 e aceito para publicação em 15.12.2010)

\section{RESUMO}

As perdas de biodiversidade não decairam nas últimas quatro décadas e as áreas protegidas, apesar de representarem uma das principais estratégias para a conservação da biodiversidade, raramente têm sido capazes de proteger sozinhas todos os habitats e espécies de interesse. No Rio Grande do Sul, 607 espécies vegetais estão em risco de extinção. Somente as arbóreo-arbustivas ameaçadas chegam a 103 espécies. Diante desse quadro, outras estratégias além das áreas protegidas devem ser pensadas com vistas a atender aos interesses da conservação de espécies ameaçadas. A conservação ex-situ, inclusive em espaços urbanos vem sendo discutida. A partir da lista das espécies da flora ameaçadas de extinção no Rio Grande do Sul foram selecionadas as espécies arbóreoarbustivas categorizadas como "Criticamente em Perigo". Revisamos as informações disponíveis para cada uma das espécies quanto aos aspectos biológicos com o propósito de incentivar o emprego destas espécies em projetos paisagísticos no meio urbano, tanto em áreas públicas quanto privadas. A seleção resultou em 15 espécies, todas com ocorrência no bioma Mata Atlântica, uma delas Gochnatia ramboi Cabrera (cambarazinho-de-rambo) também ocorre no bioma Pampa. Programas de conservação destas espécies podem ser pensados e executados nos espaços urbanos desde que integrem projetos devidamente planejados, especialmente no que se refere à integridade genética dessas espécies.

Palavras-chave: conservação ex-situ, espécies ameaçadas de extinção, ecologia urbana, biodiversidade.

\footnotetext{
1. Bióloga, Especialista em Gestão Ambiental. Mestranda do Programa de Pós-Graduação em Botânica na Universidade Federal do Rio Grande do Sul, Departamento de Botânica, Laboratório de Fitoecologia, Porto Alegre - RS. biologa.juliana@gmail.com.

2. Biólogo, Doutorando no Programa de Pós-Graduação em Ecologia e Recursos Naturais na Universidade Federal de São Carlos. São Carlos - SP. Ifperello@yahoo.com.br.
} 


\section{CONSERVATION OF ENDANGERED SPECIES FROM RIO GRANDE DO SUL BY LANDSCAPING USE}

\section{ABSTRACT}

The loss of biodiversity is not decairam in and protected areas, although they represent one of the main strategies for biodiversity conservation, rarely have been able to protect themselves all habitats and species of interest. In Rio Grande do Sul, 607 plant species are in danger of extinction. Only the tree and shrub threatened hit 103 species. On this framework, other strategies in addition to the protected areas should be prepared to meet the interests of conservation of endangered species. Ex-situ conservation, including in urban areas is being discussed. From the list of species endangered in Rio Grande do Sul were selected tree and shrub species categorized as "Critically endangered". Review the information available for each species the biological encourage the employment of these species in landscape urban projects, both in public and private areas. The selection resulted in 15 species, all with occurrence in Atlantic Forest biome, one of them Gochnatia ramboi Cabrera (cambarazinho-rambo) also occurs in Pampa biome. Conservation programmes for these species may be devised and implemented in urban areas since incorporating properly planned projects, particularly as regards genetic integrity of these species.

Keywords: ex-situ conservation, threatened of extinction species, urban ecology, biodiversity. 


\section{INTRODUÇÃO}

As metas de redução da perda da biodiversidade (CDB, 2002) não vêm sendo atingidas. Um estudo recente (BUTCHART et al., 2010) aponta que a biodiversidade seguiu diminuindo nas últimas quatro décadas, apesar do incremento de algumas iniciativas, como a ampliação da superfície de área protegida no planeta. Embora as áreas protegidas sejam uma das principais estratégias para a conservação (KATI et al., 2004), raramente são capazes de proteger sozinhas todos os hábitats e espécies de interesse. Áreas protegidas, muitas vezes, localizam-se em regiões de baixa biodiversidade enquanto alguns biomas de alta riqueza de espécies estão fora dos espaços protegidos ou mal representados por eles (TERBORGH e VAN SCHAIK, 2002). Enquanto isso, alguns fatores responsáveis pela perda direta de espécies seguem influenciando, como a fragmentação de hábitats. No caso das florestas atlânticas, $80 \%$ dos fragmentos têm área menor que $0,5 \mathrm{~km}^{2}$ e $59 \%$ dos grandes rios estão moderadamente ou intensamente fragmentados por represas e reservatórios (BUTCHART et al., 2010). Por conta dessas circunstâncias, pelo menos 1.310 espécies no planeta atualmente não estão protegidas por Unidades de Conservação (UCs) em nenhum local da sua área de distribuição, e cerca de 800 espécies correm o risco imediato de desaparecer (RODRIGUES et al., 2003; RODRIGUES et al., 2004).

A relação de espécies ameaçadas do Brasil, em sua última revisão oficial, apontou 472 espécies ameaçadas (MMA, 2008). Mas este número pode ser três vezes maior, segundo a Fundação Biodiversitas (Fundação Biodiversitas, 2009). O estado do Rio Grande do Sul abriga no seu território aproximadamente 520 espécies arbóreo-arbustivas (REITZ et al., 1983; SOBRAL et al., 2006) e destas, 103 (20\%) estão ameaçadas, sendo que 15 espécies na categoria "Criticamente em Perigo".

De acordo com a classificação da vegetação do Brasil (IBGE, 2004), a cobertura vegetal do Rio Grande do Sul está incluída em dois biomas: no Pampa, correspondente à metade sul do estado e no bioma Mata Atlântica. O bioma Pampa no Brasil é restrito ao estado do Rio Grande do Sul onde ocupa 63\% do território (IBGE, 2004). Trata-se de um bioma complexo no qual a diversidade campestre alcança aproximadamente 2.200 espécies e cuja matriz geral é formada por herbáceas com inclusões de florestas nas margens dos cursos d'água (BOLDRINI, 2009). Estima-se que mais de 250 espécies da flora deste bioma estejam ameaçadas, especialmente em decorrência da grande pressão pela qual passa para a substituição dos campos por cultivos, como soja e silvicultura (PICOLI e SCHNADELBACH, 2007; GUADAGNIN et al., 2009). O bioma Mata Atlântica, considerado

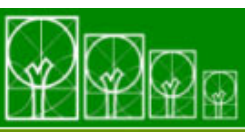

$\mathbf{S} \cdot \mathbf{B} \cdot \mathbf{A} \cdot \mathbf{U}$ Soc. Bras. de Arborização Urbana 
um dos mais biodiversos do planeta, corresponde a um mosaico de ecossistemas florestais, entre eles remanescentes da Floresta Atlântica sensu stricto, compondo ambientes integrados com a Floresta com Araucária e outros ecossistemas associados (restingas, etc.). Na metade norte do Rio Grande do Sul e nos estados de Santa Catarina e Paraná há formações campestres as quais se alternam com as florestas (OVERBECK et al., 2009). Devido a alta biodiversidade - aproximadamente 20.000 espécies para a flora - e ao grau de ameaça, a Mata Atlântica figura atualmente entre os principais hotspots do mundo. Considerando os números conhecidos de espécies em risco no Rio Grande do Sul (607) (RIO GRANDE DO SUL, 2003) e no bioma Pampa (250) (BOLDRINI, 2009), estima-se que somente na parcela sul-rio-grandense do bioma Mata Atlântica, 350 espécies da flora estejam ameaçadas.

As UCs federais, estaduais, municipais ou privadas no território do Rio Grande do Sul somam cerca de $2,6 \%$ da área do estado, mas somente $0,8 \%$ desta área são UCs de proteção integral (IBGE, 2009).

Diante desse quadro, estratégias que busquem a conservação de espécies ameaçadas além dos perímetros das áreas protegidas precisam ser consideradas. A conservação ex-situ vem sendo discutida, apontando o potencial não somente das áreas rurais agrícolas (MCNEELY e SCHERR, 2009), mas também dos espaços urbanos.

A preocupação com a qualidade do meio ambiente e a conservação dos recursos naturais, associada à qualidade de vida, leva à aproximação de distintas visões de mundo das populações. Novos conceitos de planejamento e de desenho urbano surgem, buscando atenuar problemas ambientais (GUIMARÃES e DACANAL, 2006). Embora as noções de qualidade ambiental de um lugar, em função da disponibilidade e utilização dos recursos ambientais fossem constatadas milenarmente, não envolviam uma consciência de avaliação ecológica da capacidade de suporte e de carga dos seus ecossistemas (GUIMARÃES e DACANAL, 2006). A tendência do urbanismo contemporâneo é de ampliar os valores ambientais, de modo que o paisagismo supere o valor puramente estético, valorizando-se a ecologia urbana e o planejamento ambiental (FRANCO, 1997).

A flora do Rio Grande do Sul é diversa e reúne um conjunto de espécies de valor ornamental com alto potencial para o emprego no paisagismo. No entanto, na história do paisagismo sul-rio-grandense, é notória a cópia de modelos estrangeiros. Acompanhando a arquitetura, os jardins do Brasil seguiram o ritmo das influências culturais do exterior, prevalecendo ainda hoje um modelo norte-americano (CREMA, 2007).

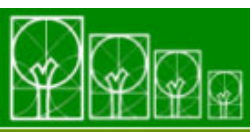

S $\cdot \mathbf{B} \cdot \mathbf{A} \cdot \mathbf{U}$ Soc. Bras. de Arborização Urbana 
Considerando a relevância e a intenção de conservar a biodiversidade em espaços urbanos, é oportuno envolver na discussão a importância do uso de espécies nativas (ISERNHAGEN et al., 2009). O emprego de espécies nativas no paisagismo visa incorporar valores de conservação, especialmente quando ele é praticado com as espécies ameaçadas. O emprego de espécies nativas nos espaços urbanos permite alcançar objetivos de educação ambiental, difundindo a identidade paisagística natural e regional. Também pode representar custos mais baixos de instalação e manutenção dos espaços e contribuir com a biodiversidade regional, tornando viáveis as populações da flora atualmente ameaçadas de extinção.

Várias tentativas de utilização de plantas nativas têm obtido sucesso em projetos de arborização urbana e na constituição de determinadas paisagens, podendo-se citar como exemplos as propostas de Lutzemberger (1985), Sanchotene (1985), Santos e Teixeira (2001), e também Burle Marx (SIQUEIRA, 2001) ao defender o emprego de espécies da família Bromeliaceae.

No entanto esta visão não é a que vigora entre os profissionais que atuam na área. Eles estão focados nas espécies mais disponíveis no mercado e seguem projetando sem considerar aspectos de conservação. Por outro, lado percebe-se também uma carência de informações sobre a nossa flora com potencial para ser empregada em projetos paisagísticos e muito mais sobre aquelas ameaçadas de extinção; muitas são espécies que reúnem alto valor ornamental, mas que ainda não são produzidas comercialmente.

Este trabalho teve como objetivo revisar informações quanto a aspectos da biologia, do cultivo e do emprego paisagístico das 15 espécies arbóreo-arbustivas constantes na lista da flora ameaçada do Rio Grande do Sul, categorizadas como "Criticamente em Perigo". Especificamente objetivamos sistematizar tais informações em tabelas com vistas a facilitar o eventual emprego das espécies em projetos paisagísticos ou de conservação ex-situ; adequar as espécies às atualizações de classificação e nomenclatura botânica e revisar as recomendações quanto ao acesso e multiplicação destas espécies, especialmente sob a ótica das implicações genéticas.

\section{MATERIAL E MÉTODOS}

A partir da lista das espécies da flora ameaçada de extinção no Rio Grande do Sul (Rio Grande do Sul, 2003) foram selecionadas as espécies arbóreo-arbustivas classificadas como "Criticamente em Perigo".

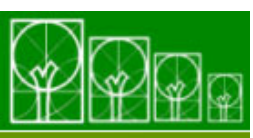

$\mathbf{S} \cdot \mathbf{B} \cdot \mathbf{A} \cdot \mathbf{U}$ Soc. Bras. de Arborização Urbana 
Foram realizadas consultas em bases de dados e artigos científicos disponíveis na internet e publicações impressas disponíveis nas bibliotecas da Universidade Federal do Rio Grande do Sul, da Pontifícia Universidade Católica do Rio Grande do Sul, da Universidade do Vale dos Sinos e do Museu de Ciências Naturais da Fundação Zoobotânica do Rio Grande do Sul. Também foram consultadas exsicatas disponíveis nos herbários regionais dessas mesmas instituições.

As informações foram organizadas em tabelas as quais reúnem aspectos sobre a biologia, o cultivo e o emprego paisagísticos das espécies. Sempre que uma determinada informação sobre a espécie não era encontrada, utilizou-se a informação disponível para seu gênero ou sua família.

Para cada espécie, foram reunidas informações sobre aspectos de sua biologia: família taxonômica, distribuição geográfica natural, bioma e ecossistema de ocorrência no Rio Grande do Sul, síndromes de polinização e dispersão, e classe sucessional ecológica. O sistema de taxonomia botânica utilizado para as famílias foi o APG III (APG, 2009).

Também foram reunidas informações sobre aspectos relacionados ao cultivo: alternativas de propagação, viabilidade germinativa das sementes, tempo de germinação, altura para o transplante, tempo para o plantio, necessidade luminosa e preferências de solo.

Quanto aos aspectos relacionados ao emprego paisagístico das espécies foram compiladas informações sobre: porte (altura máxima), persistência foliar, fenologia (períodos de floração e frutificação) e caracteres ornamentais. Os caracteres ornamentais foram identificados em fotografias e descrições encontradas em fichas de herbários. Eventualmente, ilustrações de livros também contribuíram, pois dada à raridade das espécies, nem sempre foi possível obter fotografias dos caracteres.

\section{RESULTADOS E DISCUSSÃO}

As informações sobre as espécies arbóreo-arbustivas ameaçadas do Rio Grande do Sul são escassas e fragmentadas. Constatamos que os poucos trabalhos que mencionam estas espécies são, em geral, artigos científicos com foco predominante em taxonomia.

As 15 espécies selecionadas ocorrem no bioma Mata Atlântica, uma delas Gochnatia ramboi Cabrera (cambarazinho-de-rambo) também ocorre no bioma Pampa. Taxonomicamente estão contidas em nove famílias, sendo que a Annonaceae reúne cinco espécies; Arecaceae e Lauraceae duas espécies cada uma e as demais uma espécie cada família (Tabela 1, 2 e 3).

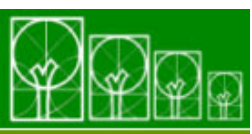

$\mathbf{S} \cdot \mathbf{B} \cdot \mathbf{A} \cdot \mathbf{U}$ Soc. Bras. de Arborização Urbana 
TABELA 1. Informações sobre aspectos da biologia das espécies arbóreo-arbustivas recomendadas para conservação ex-situ.

TABLE 1. Informations about aspects of biology of tree-shrub species recommended for ex-situ conservation.

\begin{tabular}{|c|c|c|c|c|c|c|}
\hline Família & Nome Científico & Distribuição Geográfica Natural & Bioma no RS & Ecossistema & SP SD & CS \\
\hline Annonaceae & Annona glabra L. & América e África & Mata Atlântica & FOD, RST & en hi & $P$ \\
\hline Annonaceae & Annona neosericea H. Rainer & $\begin{array}{c}\text { Brasil: Sudeste (MG, ES, SP, RJ), Sul (PR, SC, } \\
\text { RS) }\end{array}$ & Mata Atlântica & FOD, RST & en zo & NP \\
\hline Anno & Duguetia I & Brasil: Sudeste (MG, SP, RJ), Sul (PR, SC, RS) & Mata Atlântica & FOD, FE & en zo & NP \\
\hline Annor & Guatteria & $\begin{array}{l}\text { Brasil: Nordeste (BA), Centro-oeste (GO), } \\
\text { Sudeste (MG, ES,SP, RJ), Sul (PR, SC, RS) }\end{array}$ & Mata Atlântica & $\begin{array}{l}\text { FOD, RST, } \\
\text { FOM }\end{array}$ & en zo & NP \\
\hline Annonaceae & ylopia brasiliensis Sprengel & Brasil: Sudeste (MG, SP, RJ), Sul (PR, SC, RS) & Mata Atlântica & FOD, RST, FE & en si & $P$ \\
\hline Apocynaceae & Aspidosperma riedelii Müll. Arg. & América do Sul & Mata Atlântica & FE, CMP & en an & NP \\
\hline Arecaceae & Geonoma gamiova Barb. Rodr. & Brasil: Sudeste (SP, RJ), Sul (PR, SC, RS) & Mata Atlântica & FOD & en zo & NP \\
\hline Arecaceae & Geonoma schottiana Mart. & $\begin{array}{c}\text { Brasil: Sudeste (MG, ES, SP, RJ), Sul (PR, SC, } \\
\text { RS) }\end{array}$ & Mata Atlântica & $\begin{array}{l}\text { FOD, FOM, } \\
\text { RST }\end{array}$ & en zo & NP \\
\hline Asteraceae & Gochnatia ramboi Cabrera & Brasil: CO (MS), Sul (SC, RS) & $\begin{array}{c}\text { Mata Atlântica, } \\
\text { Pampa }\end{array}$ & CMP & en an & $P$ \\
\hline Lauraceae & Ocotea lobbii (Meisn.) Rohwer & $\begin{array}{c}\text { Brasil: Nordeste (BA), Sudeste (MG, ES, SP, } \\
\text { RJ), Sul (PR, SC, RS) }\end{array}$ & Mata Atlântica & $\begin{array}{l}\text { FOD, FE, } \\
\text { RST, CMP }\end{array}$ & $\begin{array}{ll}\text { en } & \text { zo } \\
(\mathrm{g}) & (\mathrm{g}) \\
\end{array}$ & NP \\
\hline Lauraceae & Persea willdenovii Kosterm. & Brasil: Sudeste (MG, SP & Mata Atlântica & $\begin{array}{l}\text { FOD, FE, } \\
\text { RST, FOM }\end{array}$ & en zo & NP \\
\hline Olacaceae & Heisteria silvianii Schwake & $\begin{array}{c}\text { Brasil: Sudeste (MG, ES, SP, RJ), Sul (PR, SC, } \\
\text { RS) }\end{array}$ & Mata Atlântica & FOD, RST & en zo & NP \\
\hline Podocarpaceae & Podocarpus sellowii Klotzsch & $\begin{array}{c}\text { Brasil: Nordeste (CE, PB, PE, SE, BA), Centro- } \\
\text { oeste (GO), Sudeste (MG, SP, RJ), Sul (PR, } \\
\text { SC, RS) }\end{array}$ & Mata Atlântica & $\begin{array}{l}\text { CMP, FOD, } \\
\text { FOM }\end{array}$ & an zo & $P$ \\
\hline Rutaceae & Esenbeckia hieronymi Engl. & Brasil: Sul (PR, SC, RS) & Mata Atlântica & FOD, RST, FE & $\begin{array}{ll}\text { en } & \text { au } \\
(\mathrm{g}) & (\mathrm{g}) \\
\end{array}$ & NP \\
\hline Vochysiaceae & $\begin{array}{l}\text { Callisthene inundata O.L.Bueno, } \\
\text { A.D.Nilson \& R.G.Magalh. }\end{array}$ & )) & Mata Atlá & OM & $\begin{array}{ll}\text { en } & \text { au } \\
(f) & (f) \\
\end{array}$ & $P$ \\
\hline
\end{tabular}


Legendas: s/i: sem informação; (g) informação referente ao gênero; (f) informação referente à família; MA: Mata Atlântica; PMP: Pampa; CMP: Formações Campestres; FE: Floresta Estacional; FOD: Floresta Ombrófila Densa; FOM: Floresta Ombrófila Mista; RST: Restinga; SP: síndrome de polinização, au: autopolinização; en: entomofilia; an: anemofilia; or: ornitofilia; qu: quiropterofilia; hi: hidrofilia; SD: síndrome de dispersão, au: autocoria; zo: endozoocoria; ep: epizoocoria; an: anemocoria; hi: hidrocoria; CS: Classe Sucessional; P: pioneira; NP: não-pioneira.

TABELA 2. Informações sobre aspectos do cultivo das espécies arbóreo-arbustivas recomendadas para conservação ex-situ.

TABLE 2. Informations about aspects of cultivation of tree-shrub species recommended for ex-situ conservation.

\begin{tabular}{cccccccc}
\hline Nome Científico & $\begin{array}{c}\text { Alternativas de } \\
\text { Propagação }\end{array}$ & $\begin{array}{c}\text { VGs } \\
\text { (dias) }\end{array}$ & $\begin{array}{c}\text { Germinação } \\
\text { (dias) }\end{array}$ & $\begin{array}{c}\text { Altura de } \\
\text { Transplante } \\
\text { (cm) }\end{array}$ & $\begin{array}{c}\text { Plantio } \\
\text { (meses) }\end{array}$ & $\begin{array}{c}\text { Necessidade } \\
\text { Luminosa }\end{array}$ & $\begin{array}{c}\text { Preferências de } \\
\text { Solos }\end{array}$ \\
\hline Annona glabra L. & $\begin{array}{c}\text { sementes, } \\
\text { estaquia }\end{array}$ & 360 & 30 a 60 & 30 & $\mathrm{~s} / \mathrm{i}$ & heliófita & úmidos \\
\hline Duguetia lanceolata A. St.Hil. & sementes & 30 & 60 a 120 & $\mathrm{~s} / \mathrm{i}$ & $\mathrm{s} / \mathrm{i}$ & heliófita & organo-argilosos, \\
secos
\end{tabular}




\begin{tabular}{|c|c|c|c|c|c|c|c|}
\hline Nome Científico & $\begin{array}{l}\text { Alternativas de } \\
\text { Propagação }\end{array}$ & $\begin{array}{l}\text { VGS } \\
\text { (dias) }\end{array}$ & $\begin{array}{l}\text { Germinação } \\
\text { (dias) }\end{array}$ & $\begin{array}{c}\text { Altura de } \\
\text { Transplante } \\
\text { (cm) }\end{array}$ & $\begin{array}{l}\text { Plantio } \\
\text { (meses) }\end{array}$ & $\begin{array}{l}\text { Necessidade } \\
\text { Luminosa }\end{array}$ & $\begin{array}{c}\text { Preferências de } \\
\text { Solos }\end{array}$ \\
\hline Esenbeckia hieronymi Engl. & sementes & $\mathrm{s} / \mathrm{i}$ & 25 a $35(g)$ & $\mathrm{s} / \mathrm{i}$ & $\mathrm{s} / \mathrm{i}$ & heliófita (g) & $\begin{array}{c}\text { argilosos, úmidos, } \\
\text { férteis }\end{array}$ \\
\hline $\begin{array}{l}\text { Callisthene inundata O.L.Bueno, } \\
\text { A.D.Nilson \& R.G.Magalh. }\end{array}$ & sementes & 5 & 2 a 5 & $\mathrm{~s} / \mathrm{i}$ & $\mathrm{s} / \mathrm{i}$ & heliófita & $\begin{array}{c}\text { rochosos, } \\
\text { arenosos, rasos, } \\
\text { úmidos }\end{array}$ \\
\hline
\end{tabular}

Legendas: s/i: sem informação; (g) informação referente ao gênero; (f) informação referente à família; VGS: Viabilidade germinativa das sementes.

TABELA 3. Informações sobre aspectos paisagísticos das espécies arbóreo-arbustivas recomendadas para conservação ex-situ.

TABLE 3. Information about aspects of landscaping of tree-shrub species recommended for ex-situ conservation.

\begin{tabular}{ccccccc}
\hline Nome Científico & Nome Popular & $\begin{array}{c}\text { Altura } \\
\text { máxima } \\
(\mathbf{m})\end{array}$ & $\begin{array}{c}\text { Persistência } \\
\text { foliar }\end{array}$ & $\begin{array}{c}\text { Período de } \\
\text { Floração }\end{array}$ & $\begin{array}{c}\text { Período de } \\
\text { Frutificação }\end{array}$ & $\begin{array}{c}\text { Caracteres } \\
\text { Ornamentais }\end{array}$ \\
\hline Annona glabra L. & araticum-do-brejo & $\leq 10$ & semi-decídua & nov a fev & out a mai & flores \\
\hline Annona neosericea H. Rainer & araticum-da-mata & $\leq 15$ & perenifólia & set a nov & dez a fev & folhas \\
\hline Duguetia lanceolata A. St.Hil. & pindabuna & $\leq 20$ & perenifólia & out a nov & mar a mai & flores e frutos \\
\hline Guatteria australis A. St.Hil. & embiú & $\leq 15$ & perenifólia & set a mar & nov & copa e frutos \\
\hline Xylopia brasiliensis Sprengel & pindaíba & $\leq 30$ & perenifólia & nov a jan & set a nov & folhas e flores \\
\hline Aspidosperma riedelii Müll. Arg. & guatambu-mirim & $\leq 6$ & semi-decídua & out a dez & ago a set & folhase flores \\
\hline Geonoma gamiova Barb. Rodr. & guaricana-rabo-de- & $\leq 4$ & perenifólia & abr a nov & jan a fev & folhas \\
\hline Geonoma schottiana Mart. & guaricana-do-brejo & $\leq 4$ & perenifólia & out a fev & mar a set & folhas \\
\hline Gochnatia ramboi Cabrera & cambarazinho-de- & $\leq 2$ & perenifólia & jan a fev & fev a mar & flores \\
\hline Ocotea lobbii (Meisn.) Rohwer & canela & $\leq 10$ & perenifólia & s/i & s/i & flores \\
\hline
\end{tabular}




\begin{tabular}{|c|c|c|c|c|c|c|}
\hline Nome Científico & Nome Popular & $\begin{array}{c}\text { Altura } \\
\text { máxima } \\
(\mathrm{m})\end{array}$ & $\begin{array}{c}\text { Persistência } \\
\text { foliar }\end{array}$ & $\begin{array}{l}\text { Período de } \\
\text { Floração }\end{array}$ & $\begin{array}{l}\text { Período de } \\
\text { Frutificação }\end{array}$ & $\begin{array}{c}\text { Caracteres } \\
\text { Ornamentais }\end{array}$ \\
\hline Persea willdenovii Kosterm. & pau-de-andrade & $\leq 25$ & semi-decídua & out a nov & jan a mar & copa \\
\hline Heisteria silvianii Schwake & casco-de-tatu & $\leq 15$ & perenifólia & ago a dez & jan a fev & frutos e flores \\
\hline Podocarpus sellowii Klotzsch & pinheiro-bravo & $\leq 3$ & perenifólia & $\mathrm{s} / \mathrm{i}$ & $\mathrm{s} / \mathrm{i}$ & folhas e frutículos \\
\hline Esenbeckia hieronymi Engl. & cutia & $\leq 8$ & semi-decídua & dez a fev & jun a ago & folhas \\
\hline $\begin{array}{l}\text { Callisthene inundata O.L.Bueno, } \\
\text { A.D.Nilson \& R.G.Magalh. }\end{array}$ & sarandi-branco & $\leq 10$ & decídua & nov & dez & folhas e flores \\
\hline
\end{tabular}

Legendas: s/i: sem informação; (g) informação referente ao gênero; (f) informação referente à família. 
As espécies representantes da família Annonaceae apresentam frutos, flores ou folhas com coloração e forma interessantes à ornamentação (Figura 1: A, B e C).

A família Arecaceae, tradicionalmente empregada no paisagismo, neste caso é representada por duas palmeiras do gênero Geonoma Willd., conhecidas como guaricanas, ambas com folhagem bastante ornamental e tolerantes a locais com baixa intensidade luminosa (Figura 1: D). A família Lauraceae também representada por duas espécies: Ocotea lobbii (Meisn.) Rohwer (canela) e Persea wildenovii Kosterm. (pau-de-andrade) espécies que apresentam grande porte sendo recomendadas para ornamentação de parques e praças - locais onde não conflitem com fiação elétrica. 


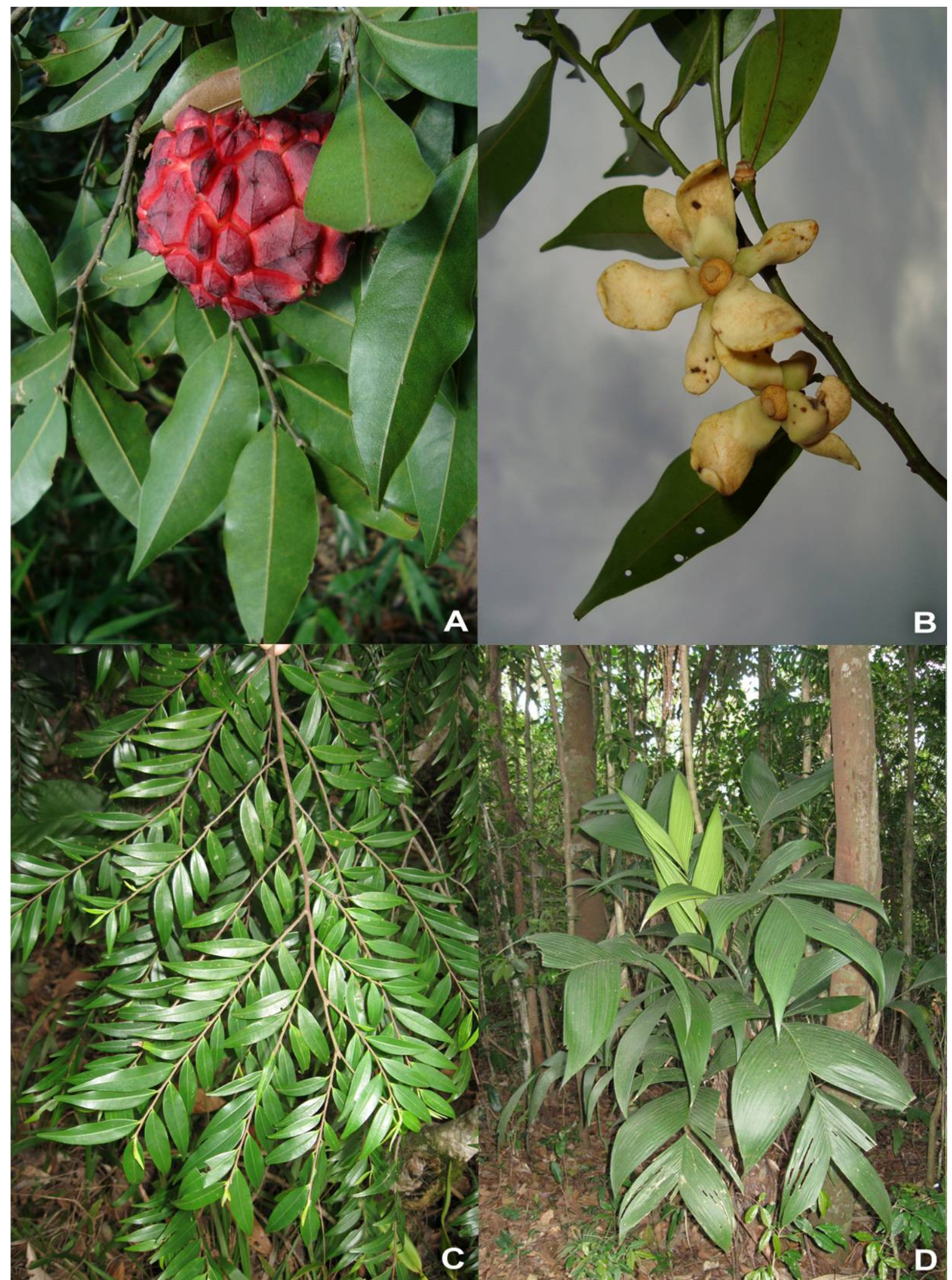

FIGURA 1. A. Duguetia lanceolata (fruto). B. Guatteria australis (flores). C. Xylopia brasiliensis (folhagem). D. Geonoma gamiova (folhagem).

FIGURE 1. A. Duguetia lanceolata (fruit). B. Guatteria australis (flowers). C. Xylopia brasiliensis (foliage). D. Geonoma gamiova (foliage).

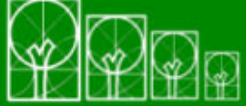

$\mathbf{S} \cdot \mathbf{B} \cdot \mathbf{A} \cdot \mathbf{U}$ Soc. Bras. de Arborização Urbana 
O arbusto Gochnatia ramboi Cabrera apresenta porte baixo e interessante à arborização de vias públicas e jardins (Figura 2: A).

A conífera Podocarpus sellowii Klotzsch ex Endl. caracterizada por folhagem ramificada (Figura 2: B), pode ter bom aproveitamento através da topiaria, técnica de poda que esculpe formas na vegetação com propósito de adorno. Da família Olacaceae, Heisteria silvianii Schwacke (casco-de-tatu) apresenta frutos de cores contrastantes (Figura 2: C).

Callisthene inundata O.L.Bueno, A.D.Nilson e R.G.Magalh. (sarandi-branco), da família Vochysiaceae, é endêmica no Rio Grande do Sul, restrita a bacia hidrográfica dos rios Taquari-Antas onde integra matas ripárias (Figura 2: D). Por conta disso, e também pela folhagem ramificada interessante a ornamentação, a espécie poderia ser empregada na proteção e ornamentação das margens de corpos d'água naturais ou artificiais no espaço urbano. As demais espécies apresentam distribuição mais ampla, incluindo outros estados do território nacional. 


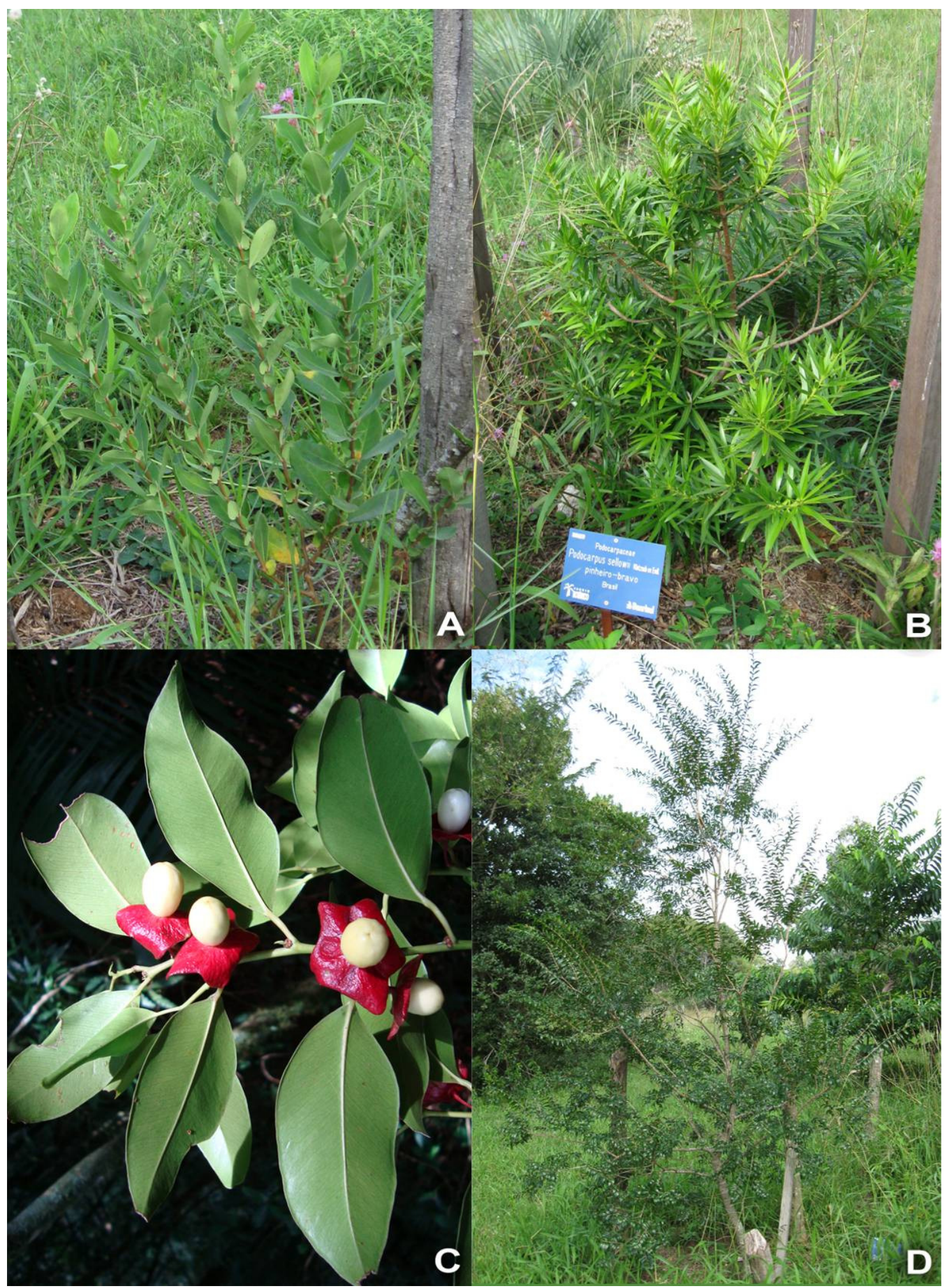

FIGURA 2. A. Gochnatia ramboi (aspecto geral). B. Podocarpus sellowii (folhagem). C. Heisteria hieronymi (fruto). D. Callisthene inundata (aspecto geral).

FIGURE 2. A. Gochnatia ramboi (general appearance). B. Podocarpus sellowii (foliage). C. Heisteria hieronymi (fruit). D. Callisthene inundata (general appearance).

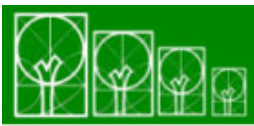

S $\cdot \mathbf{B} \cdot \mathbf{A} \cdot \mathbf{U}$ Soc. Bras. de Arborização Urbana 
Nossa revisão permitiu constatar que a atual lista de espécies da flora ameaçadas de extinção do Rio Grande do Sul necessita ser revisada quanto à nomenclatura dos táxons. Este é o caso de Persea pyrifolia (D. Don) Spreng e Rollinia sericea (R. E. Fr.) R. E. Fr.. Segundo Sobral et al. (2006), o nome Persea pyrifolia Ness e Mart., criado em 1833 e usado até recentemente, é um homônimo posterior da espécie asiática Persea pyrifolia (D. Don) Spreng, proposta em 1827, que tem prioridade de uso de acordo com o Código Internacional de Nomenclatura Botânica; assim Persea willdenovii Kosterm. é o nome que deve ser usado para a espécie americana de nome-popular "pau-de-andrade". Também, a inclusão do gênero Rollinia A. St-Hil. no gênero Annona L. (Rainer, 2007) altera a nomenclatura de Rollinia sericea (R. E. Fr.) R. E. Fr. para Annona neosericea H. Rainer (Rainer, 2007).

Para a condução da estratégia de conservação ex-situ através do uso paisagístico das espécies ameaçadas, existem alguns pontos ainda não-resolvidos, como a proibição da coleta de plantas silvestres para comercialização e as conseqüências quanto à translocação de espécies para outra região, criando o risco de cruzamento com as populações nativas, que por muito tempo ficaram isoladas a ponto de formarem variedades; existe também o risco da proliferação de apenas um determinado genoma. As espécies que se encontram ameaçadas apresentam distribuição restrita e baixa variabilidade genética (Higa e Silva, 2006).

A coleta de plantas na natureza, regulada pela Medida Provisória №. 2.186-16 de 2001, só pode ocorrer com autorização do Conselho de Gestão do Patrimônio Genético, do Ministério do Meio-Ambiente. No entanto, o cumprimento da lei esbarra nas dificuldades de fiscalização de áreas naturais e na apreciação de espécies, que podem ou não estar ameaçadas, com vista à ornamentação, aumentando a pressão de coleta.

A propagação em viveiros legalizados tende a diminuir a pressão de coletas na natureza. Em contrapartida, estas plantas correm o risco de perderem ainda mais a variabilidade genética, pois para ornamentação, os criadores/melhoristas podem priorizar (selecionar) determinadas características em detrimento de outras. Isto alteraria as freqüências gênicas da população, podendo aumentar a erosão genética, diminuindo a variabilidade.

A vulnerabilidade resultante deste estreitamento de bases genéticas pode ser evitada com cruzamentos ao acaso entre as populações para tentar transmitir toda sua herança genética (Kageyama et al., 1998). A fragmentação é uma das principais conseqüências da erosão genética, pois os remanescentes cruzam entre si. Nos viveiros, quando os indivíduos atingem a idade reprodutiva, o cruzamento preferencial pode repetir o que acontece na

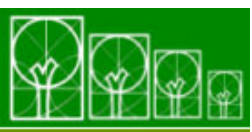

$\mathbf{S} \cdot \mathbf{B} \cdot \mathbf{A} \cdot \mathbf{U}$ Soc. Bras. de Arborização Urbana 
fragmentação de hábitats, diminuindo assim a variabilidade dos que se cruzam. Higa e Silva (2006) defendem, no entanto, que quando se busca a máxima variabilidade genética, o isolamento contra pólen externo é indesejável, aspecto que pode ser garantido no viveiro. Os autores destacam também que para atender o que vem sendo sugerido por pesquisadores do tema, um pomar de sementes visando à conservação genética de uma espécie deve ter um tamanho efetivo populacional $(\mathrm{Ne})$ igual ou superior a 150.

Em que pese todas estas implicações, o avanço da degradação dos habitats deve acentuar o risco de perda de espécies, exigindo as mais variadas e rápidas abordagens de conservação a fim de interceptar esta trajetória. A conservação ex-situ, mesmo acompanhada ainda de uma série de questionamentos com poucas respostas, pode atender esta expectativa. Portanto, a utilização para ornamentação de plantas nativas ameaçadas, poderá manter um banco genético muito maior que o de germoplasmas ou de instituições de pesquisas, sugerindo que esta estratégia pode ser exitosa, desde que devidamente planejada.

\section{CONCLUSÃO}

Todas as 15 espécies arbóreo-arbustivas enquadradas como "Criticamente em Perigo" na lista das espécies da flora ameaçada no Rio Grande do Sul apresentam potencial para emprego paisagístico, visto que reúnem um ou mais caracteres ornamentais.

Projetos paisagísticos contemplando o emprego das espécies arbóreo-arbustivas referidas neste trabalho devem priorizar municípios integrantes do bioma Mata Atlântica, pois as 15 espécies listadas têm como área natural de distribuição este bioma.

As principais implicações envolvendo o manejo das espécies sob a ótica da genética já encontram respostas entre os especialistas, não se constituindo obstáculos para o emprego das mesmas, desde que realizado com o planejamento adequado.

Também não foram identificados obstáculos importantes de outra ordem e que não recomende o emprego das espécies ameaçadas em projetos paisagísticos.

Observado o atual estado de conservação das espécies percebe-se a necessidade de que o tema tenha sua discussão ampliada para agregar novas e necessárias abordagens, já que a questão da perda de espécies requer respostas urgentes.

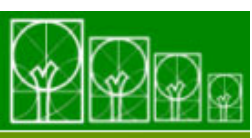

$\mathbf{S} \cdot \mathbf{B} \cdot \mathbf{A} \cdot \mathbf{U}$ Soc. Bras. de Arborização Urbana 


\section{AGRADECIMENTOS}

Ao biólogo Márcio Verdi do Inventário Florístico Florestal de Santa Catarina (IFFSC) pela concessão das fotos de Duguetia lanceolata, Guatteria australis e Heisteria silvianii.

\section{REFERÊNCIAS BIBLIOGRÁFICAS}

APG - Angiosperm Phylogeny Group. An update of the Angiosperm Phylogeny Group classification for the orders and families of flowering plants: APG III. Botanical Journal of the Linnean Society v.161, p 105-121, 2009.

Backes, P; Irgang, B. Mata Atlântica: as árvores e a paisagem. Porto Alegre: Editora Paisagem do Sul, 396 p., 2004.

Backes, P; Irgang, B. Árvores do Sul: guia de identificação e interesse ecológico. Instituto Souza Cruz, 326 p., 2002.

Backes, A; Nardino, M. Árvores, arbustos e algumas lianas nativas no Rio Grande do Sul. São Leopoldo: Editora Unisinos, 202p., 1998.

Barroso, C.M.; Delwing, A.B.; Klein, G.N.; Barros, I.B.I.; Franke, L.B. Considerações sobre a propagação e o uso ornamental de plantas raras ou ameaçadas de extinção no Rio Grande do Sul. Resumos do II Congresso Brasileiro de Agroecologia. 2007.

Boldrini, I.I. A flora dos campos do Rio Grande do Sul. Em: Pillar, V.P.; Müller, S.C.; Castilhos, Z.M.S; Jacques, A.V.A. (ed). Campos Sulinos: conservação e uso sustentável da biodiversidade. Brasília: MMA. 403p. 2009.

Butchart, S.; Walpole, M.; Collen, B.; van Strien, A.; Scharlemann, J.; Almond, R.; Baillie, J.; Bomhard, B.; Brown, C.; Bruno, J.; Carpenter, K.; Carr, G.; Chanson, J.; Chenery, A.; Csirke, J.; Davidson, N.; Dentener, F.; Foster, M.; Galli, A.; Galloway, J.; Genovesi, P.; Gregory, R.; Hockings, M.; Kapos, V.; Lamarque, J.; Leverington, F.; Loh, J.; McGeoch, M.; McRae, L.; Minasyan, A.; Morcillo, M.; Oldfield, T.; Pauly, D.; Quader, S.; Revenga, C.; Sauer, J.; Skolnik, B., Spear, D.; Stanwell-Smith, D.; Stuart, S.; Symes, A.; Tierney, M.; Tyrrell, T.; Vie, J.; Watson, R. Global Biodiversity: indicators os recent declines. Science 328, n. 5982, p 1164-1168, 2010.

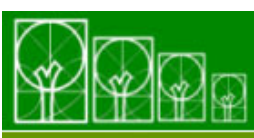

$\mathbf{S} \cdot \mathbf{B} \cdot \mathbf{A} \cdot \mathbf{U}$ Soc. Bras. de Arborização Urbana 
Calil, A.; Leonhardt, C.; Silva, V. Comportamento germinativo de sementes de guatambúmirim (Aspidosperma riedelii Müll. Arg.) Apocynaceae. Resumos do XII Encontro de Botânicos do Rio Grande do Sul, Canoas, 2004.

Calil, A.; Leonhardt, C.; Andrade, R. Comportamento germinativo de Callisthene inundata Bueno, Nilson \& Magalhães. Resumos do 51ํㅡㄹ Congresso Nacional de Botânica, Brasília, p. 89-90, 2000.

CDB - Convention on Biological Diversity. 2002. Disponível em: <http://www.cbd.int $>$. Acesso em 26/05/2010.

Stehmann, J.R.; Salino, A.; Sobral, M.; Landau, E.C.; Werneck, M.; Tamara, C.; Almeida, T. Centros de Endemismos de Plantas Vasculares na Mata Atlântica. Disponível em:

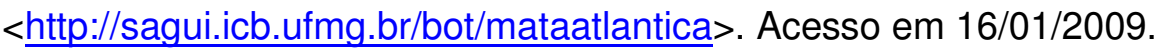

Crema, D.M.F. Paisagismo Sustentável. Jornal Construção \& Cia ed. 34, ano 03, 2007. Disponível em: <http://www.construcaoecia.com.br/conteudo.asp?ed=34\&cont=256>. Acesso em 20/04/2009.

Eltink, M.; Torres, R. B.; Ramos, E.; Galembeck, E.; Kimura, E. Guia de Árvores da Mata Atlântica. Disponível em: <http://www.ib.unicamp.br/lte/gama>. Acesso em 16/01/2009.

Fior, C.S.; Busnello, A.C.; Nilson, A.D.; Leonhardt, C.; Carneiro, A.M. Propagação sexuada de casco-de-tatu (Heisteria silvianii Schwacke - Olacaceae). Resumos do 53ํㅡㅁ Congresso Nacional de Botânica. Recife: Sociedade Botânica do Brasil, p. 13-13, 2002.

Fior, C.; Rodrigues, L.; Silva, L.; Nilson, A.; Kämpf, A. Ensaios para a propagação de Persea pyrifolia Nees et Mart. ex Nees (massaranduba). Livro de Resumos do XIII Salão de Iniciação Científica. Porto Alegre: UFRGS-PROPESQ, v. 13. p. 148-148, 2001.

Fior, C.; Leonhardt, C.; Shäffer, P.; Kämpf, A. Substrato para estaquia de Podocarpus sellowii Klotzsch ex Eichler. Resumos do IV Encontro Nacional Sobre Substrato para Planta. Viçosa: Universidade Federal de Viçosa, p. 366-366, 2004.

Franco, M. A. R. Desenho ambiental: uma introdução à arquitetura da paisagem com o paradigma ecológico. São Paulo: Annablume, 1997.

Fundação Biodiversitas. Lista da Flora Brasileira Ameaçada. 2009. Disponível em: <http://www.biodiversitas.org.br/home.htm> Acesso em 20/03/2009.

Guadagnin, D.L.; Zalba, S.; Górriz, B.; Fonseca, C.; Nebbia, A.; Cuevas, Y.; Emer, C.; Germain, P.; Wendland, E.; Perelló, L.F.C.; Bastos, M.; Sanhueza, C.; Masciadri-Bálsamo,

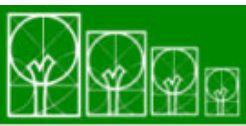

$\mathbf{S} \cdot \mathbf{B} \cdot \mathbf{A} \cdot \mathbf{U}$ Soc. Bras. de Arborização Urbana 
S.; Villalobos, A. Árvores e arbustos exóticos invasores no Pampa: questões ecológicas, culturais e sócio-econômicas de um desafio crescente. Em: Pillar, V.P.; Müller, S.C.; Castilhos, Z.M.S; Jacques, A.V.A. (ed). Campos Sulinos: conservação e uso sustentável da biodiversidade. Brasília: MMA. 403p. 2009.

Guimarães, S.T.L.; Dacanal, C. Arquitetar para viver. Educar para conservar: Faces da qualidade ambiental e da qualidade de vida na conservação do meio ambiente. Climatologia e Estudos da Paisagem v.1, p.1, 2006.

Higa, A.R.; Silva, L.D. Pomar de sementes de espécies florestais nativas. Curitiba: Fupef. 226p. 2006.

Kageyama, P.Y.; Gandara, F.B.; Souza, L.M.I. Conseqüências genéticas da fragmentação sobre populações de espécies arbóreas. Série Técnica IPEF. Genética da Fragmentação v.12, n.32, p. 65-70, 1998.

Kati, V.; Devillers, P.; Dufrene, M.; Legakis, A.; Vokou, D.; Lebrun, P. Hotspots, complementarity or representativeness? Designing optimal small-scale reserves for biodiversity conservation. Biological Conservation v.120, p.471-480. 2004.

IBGE - Instituto Brasileiro de Geografia e Estatística. Mapa de biomas e vegetação do Brasil escala 1:5.000.000. Rio de Janeiro: IBGE. 2004.

IBGE - Instituto Brasileiro de Geografia e Estatística. Mapas temáticos. 2010. Disponível em: <http://www.ibge.gov.br>. Acesso em: 25/02/2010.

Iheringia Série Botânica. Porto Alegre: Museu de Ciências Naturais, 1958-2008.

Isernhagen, I.; Bourlegat, J.; Carboni, M. Trazendo a riqueza arbórea regional para dentro das cidades: possibilidades, limitações e benefícios. Revista da Sociedade Brasileira de Arborização Urbana v.4, n.2, p.117-138, 2009.

Jonsson, M. 2008. Perda de Biodiversidade e Funcionamento dos Ecossistemas. Ecologia.info 30. Disponível em: <http://www.ecologia.info/biodiversidade.htm> Acesso em: $21 / 03 / 2009$.

Longhi, R.A. Livro das Árvores: árvores e arvoretas do sul. Porto Alegre: L\&PM, 1995.

Lorenzi, H.; Mello Filho, L.E. As plantas tropicais de R. Burle Marx. São Paulo: Nobel. 2001.

Lorenzi, H. Árvores Brasileiras: manual de identificação e cultivo de plantas arbóreas nativas do Brasil (vol.1). Nova Odessa: Instituto Plantarum. 2002a.

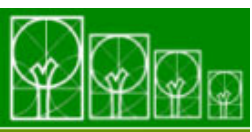

$\mathbf{S} \cdot \mathbf{B} \cdot \mathbf{A} \cdot \mathbf{U}$ Soc. Bras. de Arborização Urbana 
Lorenzi, H. Árvores Brasileiras: manual de identificação e cultivo de plantas arbóreas nativas do Brasil (vol.2). Nova Odessa: Instituto Plantarum. 2002b.

Lorenzi, H.; Souza, H.M.; Costa, J.T.M.; Cerqueira, L.S.C.; Ferreira, E. Palmeiras brasileiras e exóticas cultivadas. Nova Odessa: Instituto Plantarum. 2004.

Lorenzi, H. Plantas ornamentais no Brasil: arbustivas, herbáceas e trepadeiras. Nova Odessa: Instituto Plantarum, 2001.

Lorenzi, H.; Bacher, L.; Lacerda, M.; Sartori, S. Frutas brasileiras e exóticas cultivadas (de consumo in natura). Nova Odessa: Instituto Plantarum. 2006.

Lutzemberger, J. Ecologia: do jardim ao poder. Porto Alegre: L\&PM. 1985.

McNeely, J.A.; Scherr, S.J. Ecoagricultura: alimentação do mundo e biodiversidade. São Paulo: Senac. 2009.

MMA - Ministério do Meio Ambiente. Instrução Normativa n 6, de 23 de setembro de 2008. Disponível em:

<http://www.mma.gov.br/estruturas/179/ arquivos/179 05122008033615.pdf>. Acesso em 19/01/2009.

MMA - Ministério do Meio Ambiente. Biodiversidade Brasileira: avaliação e identificação de áreas e ações prioritárias para a conservação, utilização sustentável e repartição dos benefícios da biodiversidade nos biomas brasileiros. Brasília: MMA/SBF. 2002.

MOBOT - Missouri Botanical Garden - Vascular Tropics nomenclatural data base. Disponível em: <http://mobot.mobot.or/W3T/Search/vast.html/>. Acesso em 07/03/2010.

Overbeck, G.E.; Müller, S.C.; Fidelis, A.; Pfadenhauer, J.; Pillar, V.P.; Blanco, C.; Boldrini, I.; Both, R.; Forneck, E. Os Campos Sulinos: um bioma negligenciado. Em: Pillar, V.P.; Müller, S.C.; Castilhos, Z.M.S; Jacques, A.V.A. (ed). Campos Sulinos: conservação e uso sustentável da biodiversidade. Brasília: MMA. 403p. 2009.

Picoli, L.R.; Schnadelbach, C.V. O Pampa em Disputa: A biodiversidade ameaçada pela expansão das monoculturas de árvores. Porto Alegre: Amigos da Terra Brasil. 2007.

Rainer, H. Monographic studies in the genus Annona L. (Annonaceae): Inclusion of the genus Rollinia A. St.-Hil. Annalen des Naturhistorischen Museums in Wien v. 108B, p. 191-205, 2007

Reitz, R.; Klein, R.M.; Reis, A. Projeto Madeira do Rio Grande do Sul. Sellowia v. 34 e 35, p. 1-525, 1983.

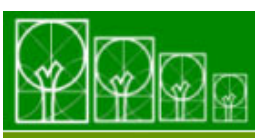

$\mathbf{S} \cdot \mathbf{B} \cdot \mathbf{A} \cdot \mathbf{U}$ Soc. Bras. de Arborização Urbana

REVSBAU, Piracicaba - SP, v.5, n.4, p.01-21, 2010 
Rodrigues, A.; Andelman, S.; Bakarr, M.; Boitani, L.; Brooks, T.; Cowling, R.; Fishpoo, L.; Fonseca, G.; Gaston, K.; Hoffmann, M.; Long, J.; Marquet, P.; Pilgrim, J.; Pressey, R.; Schipper, J.; Sechrest, W.; Stuart, S.; Underhill, L.; Waller, R.; Watts, M.; Yan, X. 2003. Global Gap Analysis. Towards a representative network of protected areas. Advances in Applied Biodiversity Science 5. Washington DC: Conservation International, 98p. 2003.

Rodrigues, A.; Andelman, S.; Bakarr, M.; Boitani, L.; Brooks, T.; Cowling, R., Fishpoo, L.; Fonseca, G.; Gaston, K.J., Hoffmann, M., Long, J., Marquet, P.; Pilgrim, J.; Pressey, R.; Schipper, J.; Sechrest, W.; Stuart, S.; Underhill, L.; Waller, R.; Watts, M.; Yan, X. Effectiveness of the global protected area network in representing species diversity. Nature 428, p. 640-643, 2004.

Rio Grande do Sul. 2008. Projeto RS BIODIVERSIDADE. Disponível em: $<$ http://www.biodiversidade.rs.gov.br>. Acesso em: 28/05/2008.

Rio Grande do Sul. Decreto estadual no 42.099 de 31 de dezembro de 2002 (Lista Oficial da Flora Ameaçada de Extinção do Rio Grande do Sul). Diário Oficial do estado do Rio Grande do Sul, n 1, 2003.

Salomão, A.N.; Sousa-Silva, J.; Davide, A.; Gonzáles, S.; Torres, R.A.A.; Wetzel, M.; Firetti, F.; Caldas, L.S. Germinação de Sementes e Produção de Mudas de Plantas do Cerrado. Brasília: Rede de sementes do Cerrado, 2003.

Sanchotene, M.M.C. Frutíferas nativas úteis à fauna na arborização urbana. Porto Alegre: Feplam, 1985.

Santos, N.R.Z.; Teixeira, I.F. Arborização de vias públicas: ambiente x vegetação. Santa Cruz do Sul: Instituto Souza Cruz, 2001.

Siqueira, V.B. Burle Marx. São Paulo: Cosac e Naify, 2001.

Sobral, M.; Jarenkow, J.A.; Brack, P.; Irgang, B.; Larocca, J.; Rodrigues, R.S. Flora arbórea e arborescente do Rio Grande do Sul, Brasil. São Carlos: RiMA/Novo Ambiente, 2006.

Terborgh, J.; Chaik, C. van. 2002. Por que o Mundo Necessita de Parques. Em: Terborgh, J.; Schaik, C. van; Davenport L.; Rao, M. (org). Tornando os Parques Eficientes: estratégias para a conservação da natureza nos trópicos. Pág. 25. Curitiba: Editora da Universidade Federal do Paraná. Fundação O Boticário, 2002. 